\title{
Inertial Actuator with Virtual Mass for Active Vibration Control
}

\author{
Qibo Mao \\ School of Aircraft Engineering, Nanchang HangKong University, 696 South Fenghe Avenue, Nanchang, CN- \\ 330063, P. R. China.
}

\section{Shenquan Li}

School of Hydraulic, Energy and Power Engineering, Yangzhou University, 196 West Huayang Road, Yangzhou CN-225127, P.R.China.

\author{
Shizuo Huang \\ School of Aircraft Engineering, Nanchang HangKong University, 696 South Fenghe Avenue, Nanchang, CN- \\ 330063, P. R. China.
}

(Received 6 January 2020; accepted 13 July 2020)

\begin{abstract}
Inertial actuators (IAs) are often used as control units in active noise and vibration control systems. It is wellknown that the IA's natural frequency should be far below that of the structure under control to ensure good stability margins. However, under normal circumstances, an IA with low natural frequency either increases the additional weight or causes unwanted static displacement of the IA's proof-mass. In this study, an IA with virtual mass is presented to reduce the IA's natural frequency without changing its physical design. The virtual mass of the IA is realized by using the proof-mass acceleration feedback as a local loop within the IA. Thus, the IA's natural frequency can be shifted to low frequency for active control application. The proposed IA with virtual mass is then applied to actively control a clamped beam's vibration based on the velocity feedback control system. The experimental results show that the stability of the control system and the control performance can be improved significantly as the IA's natural frequency is reduced with virtual mass.
\end{abstract}

\section{NOMENCLATURE}

$a_{a}(\omega)$ Complex amplitude of the proof-mass acceleration of the IA

Bl Electromagnetic transduction coefficient

$C_{a} \quad$ Suspension damping coefficient of the IA

$f_{n} \quad$ Natural frequency of the IA

$g_{p} \quad$ Positive or negative acceleration feedback

$G_{c} \quad$ Open-loop FRF of the control path

$h \quad$ Fixed feedback control gain

$i(\omega) \quad$ Complex amplitude of the current flowing through the coil

$I(t) \quad$ Current flowing through the coil in time-domain

$j \quad$ Imaginary unit

$K_{a} \quad$ Suspension stiffness of the IA

$L_{e} \quad$ Inductance of the coil

$M_{a} \quad$ proof mass of the IA

$M_{v} \quad$ Virtual mass

$R_{e} \quad$ Resistance of the coil

$T_{a} \quad$ Blocked force per unit input voltage

$v_{a}(\omega)$ Complex amplitude of the proof-mass velocity of the IA

$v_{d}(\omega)$ Error sensor location velocity due to primary force

$v_{s}(\omega)$ Complex amplitude of base velocity of the IA

$V_{s}(\omega)$ Complex amplitude of voltage applied to the coil

$V_{\text {in }}(\omega)$ Complex amplitude of input voltage of the velocity feedback loop $x_{a}(\omega)$ Complex amplitude of proof-mass displacement of the IA

$x_{s}(\omega)$ Complex amplitude of base displacement of the IA

$X_{a}(t)$ Proof-mass displacement of the IA in time-domain

$X_{s}(t)$ Base displacement of the IA in time-domain

$Z_{a} \quad$ Undriven mechanical impedance of the IA

$\omega \quad$ Circular frequency

\section{INTRODUCTION}

Inertial actuators (IAs) are efficient control units in active noise and vibration control systems for reducing sound and vibration in the low frequency range. ${ }^{1-9}$ The main advantage of the use of IAs is that there is no requirement of any other structure to react off. ${ }^{1,2,5}$ At frequencies above the natural frequency of the IA, it can effectively generate a constant actuation force and in-phase with the input driving voltage. ${ }^{9}$ Consequently, when an accelerometer with integrated output is collocated at primary structure, the IA can be used to perform velocity feedback control, which reduces the structure vibration by means of active damping. ${ }^{10-12}$

At frequencies lower than the IA's natural frequency, the force produced by the IA has $180^{\circ}$ phase shift, thus a negative damping effect occurs, which may lead to instabilities in the feedback control system. ${ }^{4,9}$ It means that the IA's natural frequency should be far below that of the structure under control in order to ensure good stability margins and improve 
its control performance. ${ }^{9,13}$ However, the IA with low natural frequency normally increases the unwanted weight of the IA proof-mass or causes unwanted static displacement of the proof-mass with the lower stiffness of the IA spring.

For velocity feedback control system by using IA with collocated accelerometer sensor, there are two approaches to improve the control performance limitation of the IAs. The first approach is to develop the new type IAs which have much lower natural frequency. For examples, Olaru et al. ${ }^{14}$ used a differential magnetic spring to replace the classical elastic suspensions in IAs. Braghin et al. ${ }^{15}$ introduced a new design method for the supporting structure of the magnetostrictive spring, thus the IA can extend its lower frequency working ranges by reduction of its natural frequency. Kras and Gardonio ${ }^{16}$ imposed a flywheel element into the traditional IA, thus the IA's natural frequency moved to lower frequency. This new type flywheel inertial actuator has been experimentally verified. ${ }^{17}$ Zilletti $^{18}$ presented a theoretical study on an IA with an inerter element. It is found that the IA's natural frequency can be shifted down because of the inerter. The second approach is to modify the velocity feedback control loop to make the control system perfectly collocated. For examples, Benassi and Elliott ${ }^{19}$ imposed additional internal displacement feedback loop to overcome the static deflection problem of the IA with very low natural frequency. ${ }^{19}$ Diaz et al. ${ }^{20}$ extended this approach for the control of human-induced vibration of pedestrian structures. Rohlfing et al. ${ }^{21}$ presented a new modified velocity feedback loop for IAs by adding an additional compensator filter. Diaz and Reynolds ${ }^{22}$ imposed a feed-through term into the velocity feedback controller, thus a robust control system has been designed by using the IA as control unit.

Recently, an IA, with a proof-mass acceleration feedback used as the tuneable vibration absorber, has been proposed and experimentally verified. ${ }^{23}$ The IA's natural frequency can be moved to high or low frequency under positive or negative proof-mass acceleration gains, which can produce virtual mass effect. In this study, the IA with virtual mass is extended for active control application. Different to preview studies, the proposed control system includes an IA proof-mass acceleration feedback loop and a velocity feedback loop, as presented in Fig. 1. The use of a proof-mass acceleration feedback loop has the effect of producing the virtual mass effect, thus the IA's natural frequency can be reduced. This allows the velocity feedback loop with collocated integrated accelerometer output on the vibrating structure has higher feedback control gain and hence higher active damping.

The remainder of this study is organized as follows. In Section 2, the theoretical mathematical model for the IA with virtual mass by using acceleration feedback is presented. In Section 3, the tuning capability, and the blocked force response of the IA by using proof-mass acceleration feedback are experimentally assessed. Section 4 presents the velocity feedback control experiments by using proposed IA in a clampedclamped beam. Finally, some useful conclusions are provided in Section 5.

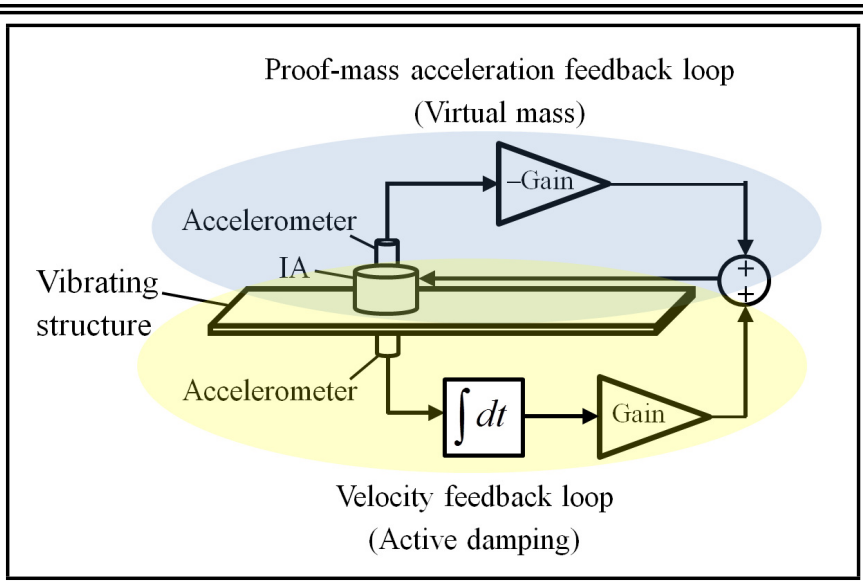

Figure 1. Schematic of the control system for IA with virtual mass.

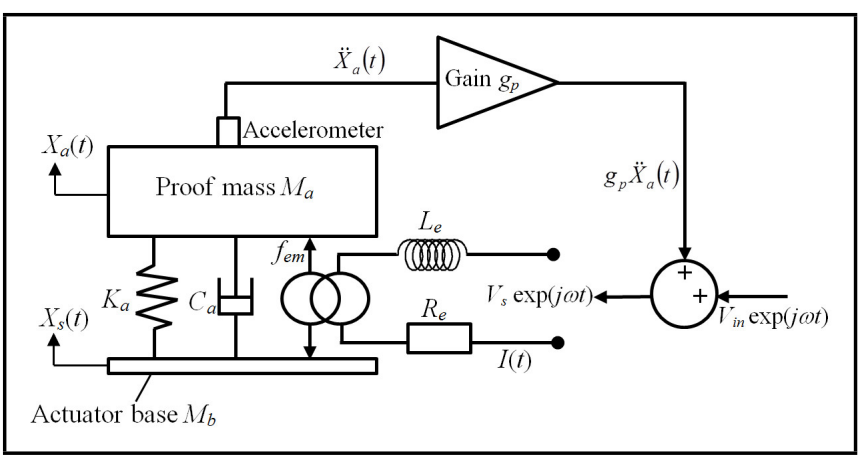

Figure 2. Schematic of the IA with virtual mass based on proof-mass acceleration feedback.

\section{THE IA WITH VIRTUAL MASS BY USING PROOF-MASS ACCELERATION FEEDBACK}

A generic schematic for an IA, with virtual mass by using proof-mass acceleration feedback considered in this study, is presented in Fig. 2. An accelerometer, which was positioned at the IA proof-mass, was used as the error sensor. This means that the feedback signal is the proof-mass acceleration, which was used to shift the IA's natural frequency.

The IA shown in Fig. 2 consisted of a proof mass $M_{a}$, mounted on a damping $C_{a}$ and a suspension of stiffness $K_{a}$ in parallel. It was assumed that the actuator base mass $M_{b}$ was very small and thus could be neglected. A linear lumped parameter model was used in this study assuming the time harmonic vibration motion of the form $\exp (j t)$, where $\omega$ was the circular frequency and $j$ was imaginary unit. Two differential equations were used to describe the IA's coupled electromechanical behaviour. The governing equation of motion of the proof mass $M_{a}$, and the equation of the electrical circuit of the IA presented in Fig. 2 was expressed as ${ }^{6}$

$$
\begin{array}{r}
M_{a} \ddot{X}_{a}(t)+C_{a}\left[\dot{X}_{a}(t)-\dot{X}_{s}(t)\right]+K_{a}\left[X_{a}(t)-X_{s}(t)\right] \\
=B l \cdot I(t) ;
\end{array}
$$

$L_{e} \dot{I}(t)+R_{e} I(t)=V_{s} \exp (j \omega t)-B l \cdot\left[\dot{X}_{a}(t)-\dot{X}_{s}(t)\right] ;$

where $B l$ was the electromagnetic transduction coefficient of 
the IA. $X_{a}(t)$ was the proof-mass displacement and $X_{s}(t)$ was the base displacement. $I(t)$ was the current flowing through the coil. () represented the derivative with respect to time. $R_{e}$ and $L_{e}$ were the resistance and inductance of the coil. $V_{s}$ was the amplitude of the voltage applied to the coil.

For harmonic vibration of the form $\exp (j t)$, we set $X_{a}(t)$, $X_{s}(t)$ and $I(t)$ in Eqs.(1) and (2) as

$$
\begin{gathered}
I(t)=i(\omega) \exp (j \omega t) ; \\
X_{a}(t)=x_{a}(\omega) \exp (j \omega t) ; \\
X_{s}(t)=x_{s}(\omega) \exp (j \omega t) ;
\end{gathered}
$$

where $i(\omega), x_{a}(\omega)$ and $x_{s}(\omega)$ were the complex amplitude of the electric current, the proof-mass displacement and the base displacement, respectively.

From Eq. (3a), the following equations can be obtained

$$
\begin{gathered}
\dot{I}(t)=j \omega \cdot i(\omega) \exp (j \omega t)=j \omega \cdot I(t) ; \\
\dot{X}_{a}(t)=j \omega \cdot x_{a}(\omega) \exp (j \omega t)=v_{a}(\omega) \exp (j \omega t) ; \\
\ddot{X}_{a}(t)=-\omega^{2} \cdot x_{a}(\omega) \exp (j \omega t)=a_{a}(\omega) \exp (j \omega t) ; \\
\dot{X}_{s}(t)=j \omega \cdot x_{s}(\omega) \exp (j \omega t)=v_{s}(\omega) \exp (j \omega t) ;
\end{gathered}
$$

where $v_{a}(\omega)=j \omega \cdot x_{a}(\omega)$ and $a_{a}(\omega)=-\omega^{2} \cdot x_{a}(\omega)$ were the complex amplitude of the proof-mass velocity and acceleration, respectively. $v_{s}(\omega)=j \omega \cdot x_{s}(\omega)$ was the complex amplitude of the base velocity.

For brevity, the frequency dependence of the complex amplitudes will be omitted in the remaining part of the paper. By substituting Eqs. (3) and (4), into Eqs.(1) and (2), we got

$$
\begin{gathered}
M_{a} a_{a}+C_{a}\left(v_{a}-v_{s}\right)+K_{a}\left(x_{a}-x_{s}\right)=B l \cdot i ; \\
j \omega L_{e} i+R_{e} i=V_{s}-B l \cdot\left(v_{a}-v_{s}\right) .
\end{gathered}
$$

From Fig.2, it can be found that $V_{s}$ was the sum of the proof-mass acceleration feedback voltage and an additional input voltage $V_{i n}$, such as

$$
V_{s}=V_{i n}+a_{a} \cdot g_{p}
$$

where $g_{p}$ was the positive or negative acceleration feedback.

Substituting Eq. (7) into Eq. (6), the current in Eq. (6) was rewritten as

$$
i=\frac{V_{i n}+a_{a} \cdot g_{p}-B l \cdot\left(v_{a}-v_{s}\right)}{j \omega L_{e}+R_{e}} .
$$

Substituting Eq. (8) into Eq. (5), we got

$$
\begin{aligned}
& C_{a} v_{s}+\left(K_{a}+\frac{j \omega(B l)^{2}}{j \omega L_{e}+R_{e}}\right) x_{s}+\frac{B l}{j \omega L_{e}+R_{e}} V_{i n} \\
& =\left(M_{a}-\frac{B l \cdot g_{p}}{j \omega L_{e}+R_{e}}\right) a_{a}+\left(C_{a}+\frac{(B l)^{2}}{j \omega L_{e}+R_{e}}\right) v_{a}+K_{a} x_{a} \\
& =\left(M_{a}+M_{v}\right) a_{a}+\left(C_{a}+\frac{(B l)^{2}}{j \omega L_{e}+R_{e}}\right) v_{a}+K_{a} x_{a} ;
\end{aligned}
$$

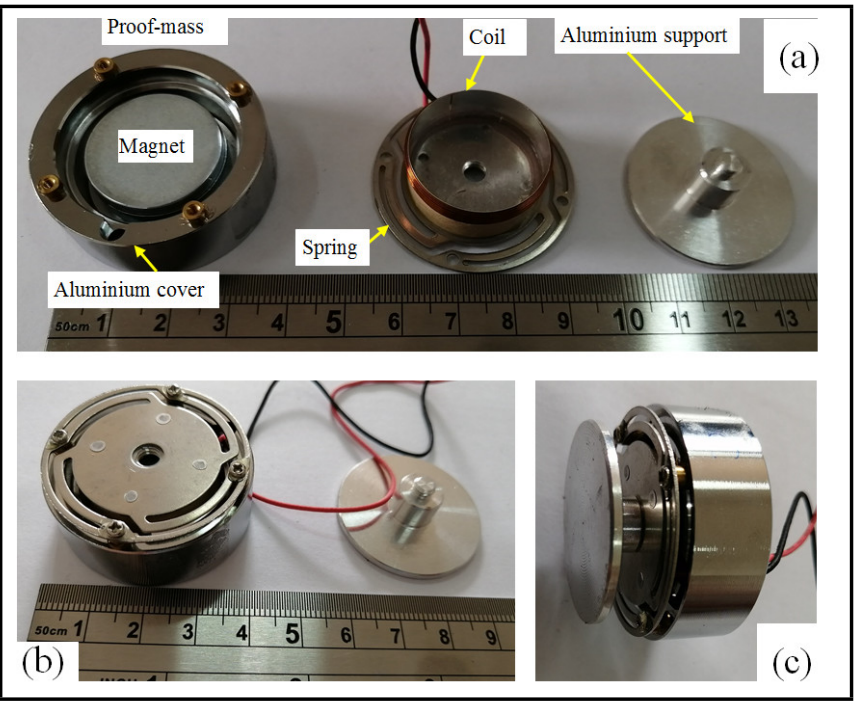

Figure 3. Picture of the home-made IA (a) before assembly; (b) partly assembly; (c) after assembly.

where $M_{v}=-\frac{B l \cdot g_{p}}{j \omega L_{e}+R_{e}}$.

From Eq. (9), it can be found that the term $M_{v}$ influences the IA proof mass, thus $M_{v}$ was denoted as the virtual mass. ${ }^{23}$ According to Refs., ${ }^{23,24}$ the term $j L_{e}$ in $M_{v}$ can be neglected because the coil inductance $L_{e}(0.34 \mathrm{mH}$ in this study) in IA was normally sufficiently small, ${ }^{24}$ therefore the value of $L_{e}$ (about 0.21 at $100 \mathrm{~Hz}$ ) at low frequency was much smaller than $R_{e}$ (4.4 in this study). Clearly, the IA's natural frequency could be shifted down by adding positive $M_{v}$ when the negative acceleration feedback was used.

From Eq. (9), the proof-mass acceleration was expressed as

$$
a_{a}=-\omega^{2} \frac{\left(C_{a}+\frac{K_{a}}{j \omega}+\frac{(B l)^{2}}{j \omega L_{e}+R_{e}}\right) v_{s}+\frac{B l}{j \omega L_{e}+R_{e}} V_{i n}}{-\omega^{2}\left(M_{a}+M_{v}\right)+j \omega\left(C_{a}+\frac{(B l)^{2}}{j \omega L_{e}+R_{e}}\right)+K_{a}} .
$$

From Eq. (10), the force generated by an IA, $f_{a}$, was expressed as the sum of the component due to its input voltage $V_{i n}$, and that due to the imposed velocity on its base $v_{s}$, we got

$$
f_{a}=M_{a} a_{a}=T_{a} V_{i n}+Z_{a} v_{s}
$$

with

$$
T_{a}=\frac{-\omega^{2} M_{a} \frac{B l}{j \omega L_{e}+R_{e}}}{-\omega^{2}\left(M_{a}+M_{v}\right)+j \omega\left(C_{a}+\frac{(B l)^{2}}{j \omega L_{e}+R_{e}}\right)+K_{a}} ;
$$

$$
Z_{a}=\frac{-\omega^{2} M_{a}\left(C_{a}+\frac{K_{a}}{j \omega}+\frac{(B l)^{2}}{j \omega L_{e}+R_{e}}\right)}{-\omega^{2}\left(M_{a}+M_{v}\right)+j \omega\left(C_{a}+\frac{(B l)^{2}}{j \omega L_{e}+R_{e}}\right)+K_{a}}
$$

where $T_{a}$ was the blocked force per unit input voltage and $Z_{a}$ was the IA's undriven mechanical impedance. ${ }^{24}$

\section{EXPERIMENTAL ASSESSMENT OF THE IA WITH VIRTUAL MASS}

As discussed in Section 2, the virtual mass based on a proofmass acceleration feedback loop can be used to shift the IA's 


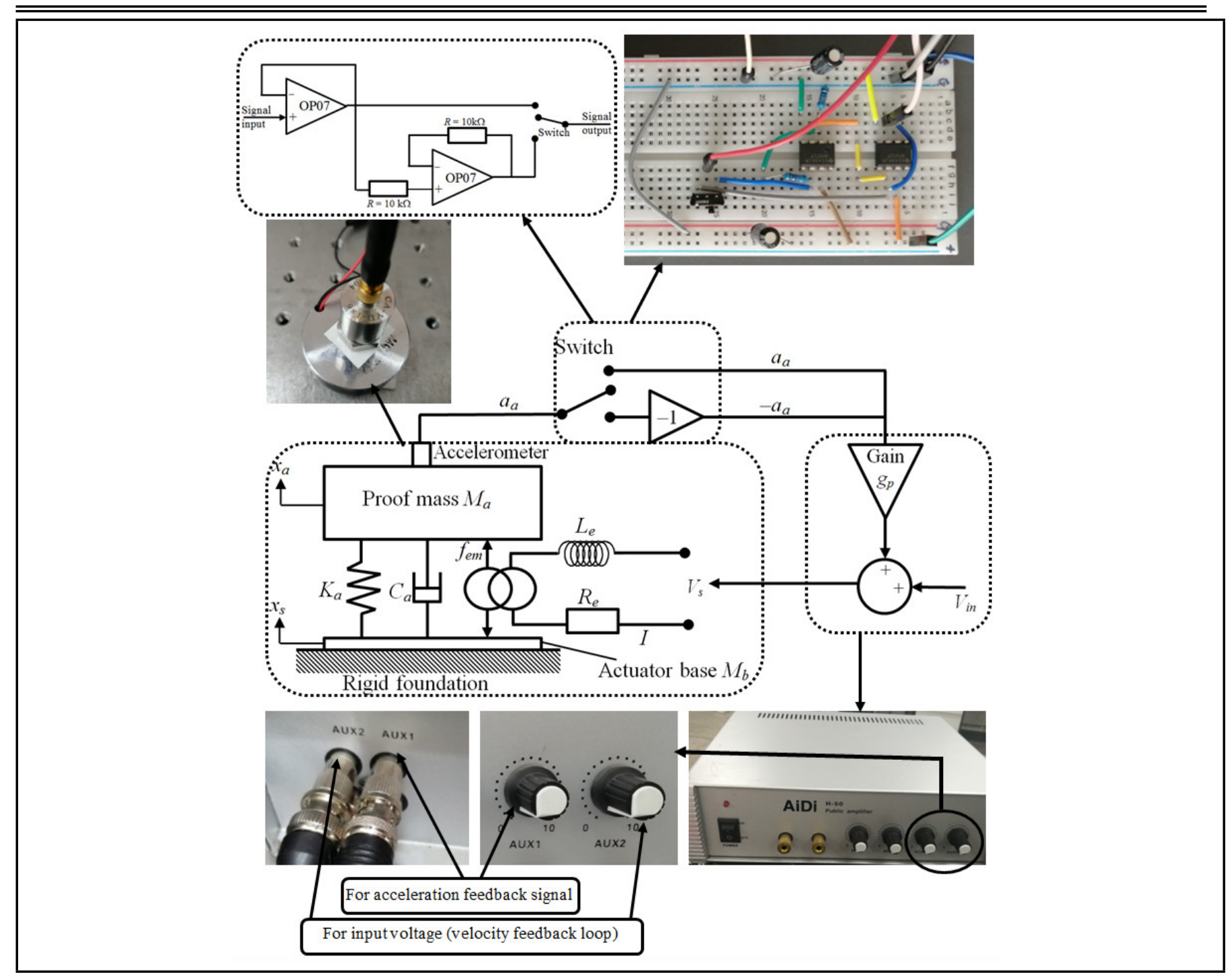

Figure 4. Schematic representation of the experimental setup to measure the blocked force response of the IA with acceleration feedback.

Table 1. The physical parameters of the low-cost home-made IA.

\begin{tabular}{||l|l|l|l||}
\hline Parameter & Symbol & Value & Unit \\
\hline Proof mass & $M_{a}$ & $82 \times 10^{-3}$ & $\mathrm{~kg}$ \\
Support mass & $M_{b}$ & $6.3 \times 10^{-3}$ & $\mathrm{~kg}$ \\
Suspension stiffness & $K_{a}$ & $39.1 \times 10^{3}$ & $\mathrm{~N} / \mathrm{m}$ \\
Suspension damping coefficient & $C_{a}$ & 1.2 & $\mathrm{Ns} / \mathrm{m}$ \\
Coil resistance & $R_{e}$ & 4.4 & \\
Coil inductance & $L_{e}$ & $0.34 \times 10^{-3}$ & $\mathrm{H}$ \\
Electromagnetic transduction coefficient & $B l$ & 4.8 & $\mathrm{~N} / \mathrm{A}$ \\
Natural frequency & $f_{n}$ & 109.9 & $\mathrm{~Hz}$ \\
\hline
\end{tabular}

natural frequency. Before applying the proposed IA as control unit in active vibration control system, it was necessary to examine the tuning ability of the IA's natural frequency. A lowcost home-made IA was used in this study, as shown in Fig. 3. The physical parameters of this IA are presented in Table 1.

The blocked force response of the IA was experimentally measured when the actuator base was mounted on a rigid foundation, it means that the base velocity $v_{s}=0$. From Eq. (11), it can be found that blocked force response was $T_{a}=\frac{f_{a}}{V_{i n}}=$ $\frac{M_{a} a_{a}}{V_{i n}}$ for $v_{s}=0$. An experimental setup presented in Fig. 4 was used to measure the blocked force response $T_{a}$ of the IA. A CA-YD-186 accelerometer (with weight of $28 \mathrm{~g}$ ), which is attached on the IA proof-mass, was used to sense the proof-mass acceleration. A COINV dynamic signal analyzer was used to create the excitation signal and acquire the frequency response functions (FRFs) between input voltage and proof-mass accelerations. A simple switch circuit, by using OP07 Voltage Operational Amplifier (by Analog Devices Inc.), was designed to feedback the negative or positive acceleration signal. Then a power amplifier with two input channels was used to input the excitation signal and acceleration feedback signal. It means that the sum of the acceleration signal and the excitation signal were used to drive the IA. The gain of each channel of the power amplifier was independent tunable, as presented in Fig. 4. By changing the gain of the acceleration feedback channel, the blocked force responses $T_{a}$, which equal to the measured acceleration signals multiplied the value of proof mass, under different acceleration feedback gains was determined. All measurements were performed at small input voltage level so that the mechanical behavior of the IA is effectively linear.

Figures 5 and 6 show the measured and calculated blocked force response of the IA under different feedback gains of the acceleration signal, respectively. As presented in Fig. 5, because of the accelerometer mass, the IA's natural frequency without feedback control $\left(g_{p}=0\right)$ was about $99.5 \mathrm{~Hz}$. When a negative acceleration feedback gain $g_{p}$ was applied, the IA's natural frequency was able to move to a much lower frequency, 


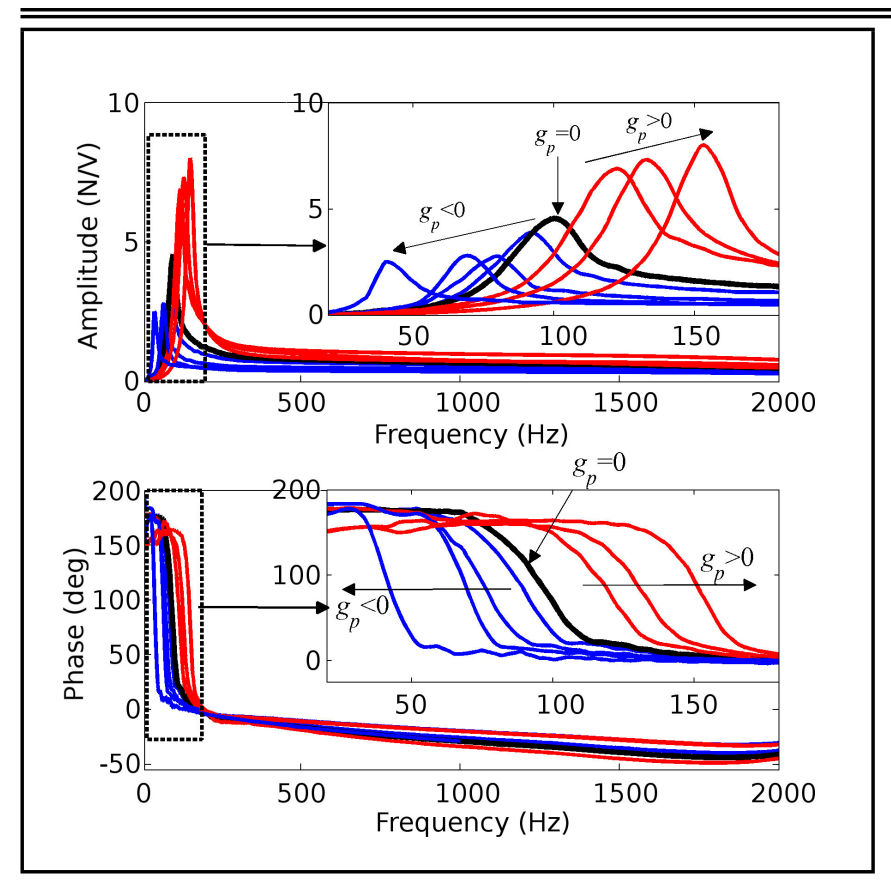

Figure 5. The measured blocked force response of the IA with different gains $g_{p}$.

and the peak amplitudes of blocked force responses were decreased as negative gain increased. Alternatively, the IA's natural frequency was moved to high frequency with positive gains. From Figs. 5 and 6, it can also be found that the phases of the blocked force responses shifted to $180^{\circ}$ and the amplitudes fell off below the natural frequency, as expected. Above the IA's natural frequency, the amplitudes and phases of the blocked force responses were reasonably flat up.

The measured and numerical calculated open-loop frequency response in Nyquist format, from IA voltage input to signal output from the accelerometer on proof-mass, is also shown in Fig. 7. From Fig. 7, it can be found that the ideal IA was unconditionally stable with negative acceleration feedback gain because of the instability point $(-1,0)$ is excluded from the loop. However, the measurement Nyquist plot indicates the feedback loop is not unconditionally stable due to the dynamic of the power amplifier. The natural frequency of the IA used in this study was reduced from $99.5 \mathrm{~Hz}$ to $40 \mathrm{~Hz}$ when the negative acceleration feedback gain $g_{p}$ was tuned from 0 to -3.5 . The calculated and experimental results presented in Figs. 5-7 confirm that the IA's natural frequency can be reduced significantly by using negative proof-mass acceleration feedback loop as virtual mass.

\section{EXPERIMENTAL INVESTIGATION OF VELOCITY FEEDBACK CONTROL}

\subsection{Experimental setup}

In this section, with an example of an aluminium clampedclamped beam, the control performances of the proposed IA with virtual mass as control actuator were investigated for a velocity feedback control system. The basic idea is shown in Fig. 1 and the experimental setup is shown in Fig. 8. The IA with virtual mass mounted near one clamped end was used to control the beam vibration. The size and mass of beam were
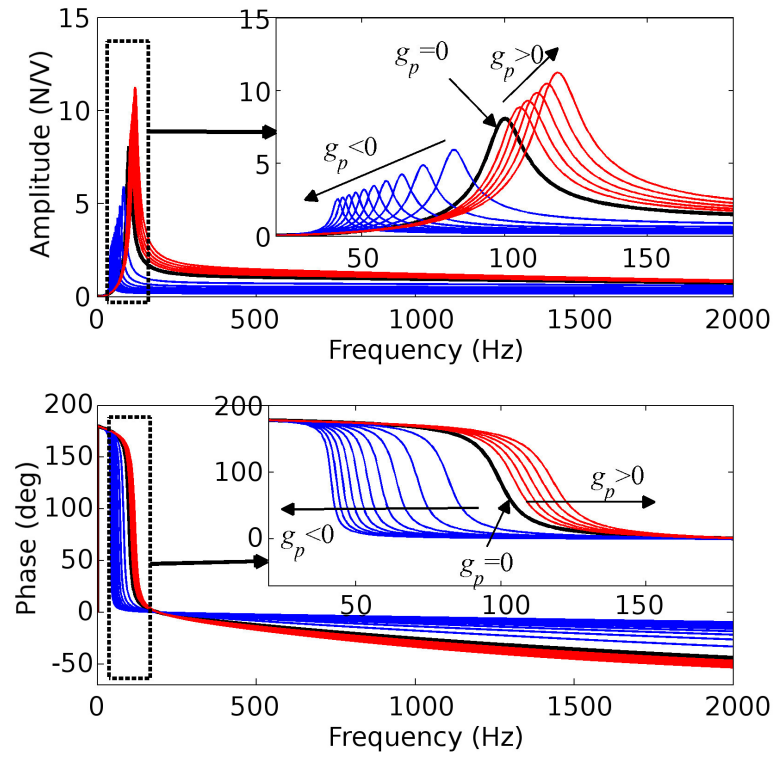

Figure 6. The calculated blocked force response of the IA with different gains $g_{p}$.

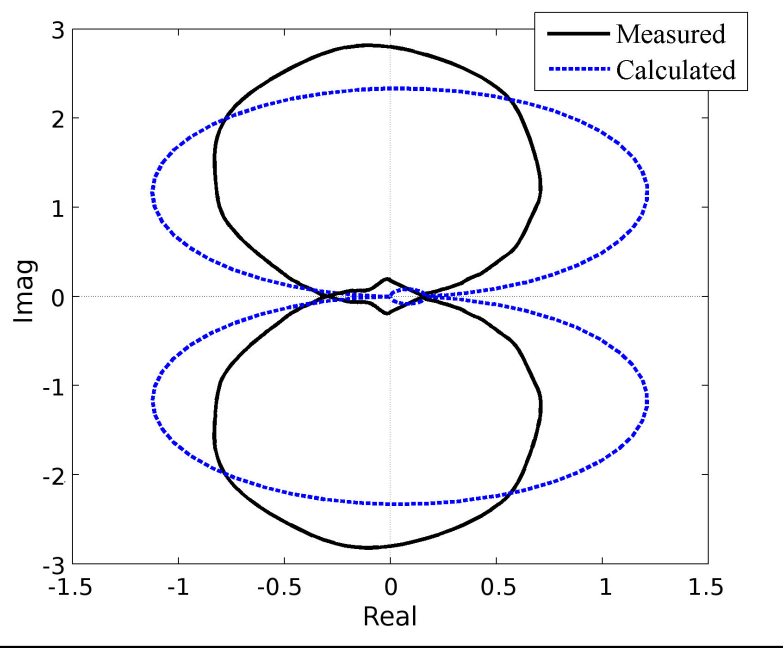

Figure 7. The measured and calculated Nyquist plots for IA.

$580 \times 50 \times 7 \mathrm{~mm}$ and $550 \mathrm{~g}$, respectively. The beam was excited by a sweep-sine signal with frequency ranges from $0-800 \mathrm{~Hz}$ by using another IA near another clamped end. The mass of the control IA including accelerometer on the proof-mass was $100 \mathrm{~g}$, which equals to $18.18 \%$ weight of the beam. Another accelerometer was mounted at centre of control IA location (on the bottom of the beam), its integrated output was used as sensor signal in velocity feedback loop, as shown in Fig. 8.

\subsection{Stability and control performance}

According to Refs., $5,6,18,24$ the velocity feedback control system was modelled in terms of the classic disturbance rejection feedback block diagram, as presented in Fig. 9. Since the velocity feedback controller used in this study was not modalbased, it means that the frequency response of the control loop was sufficient to analyze the stability and the state-space model is not needed. From Fig. 9, it can be found that the velocity at 


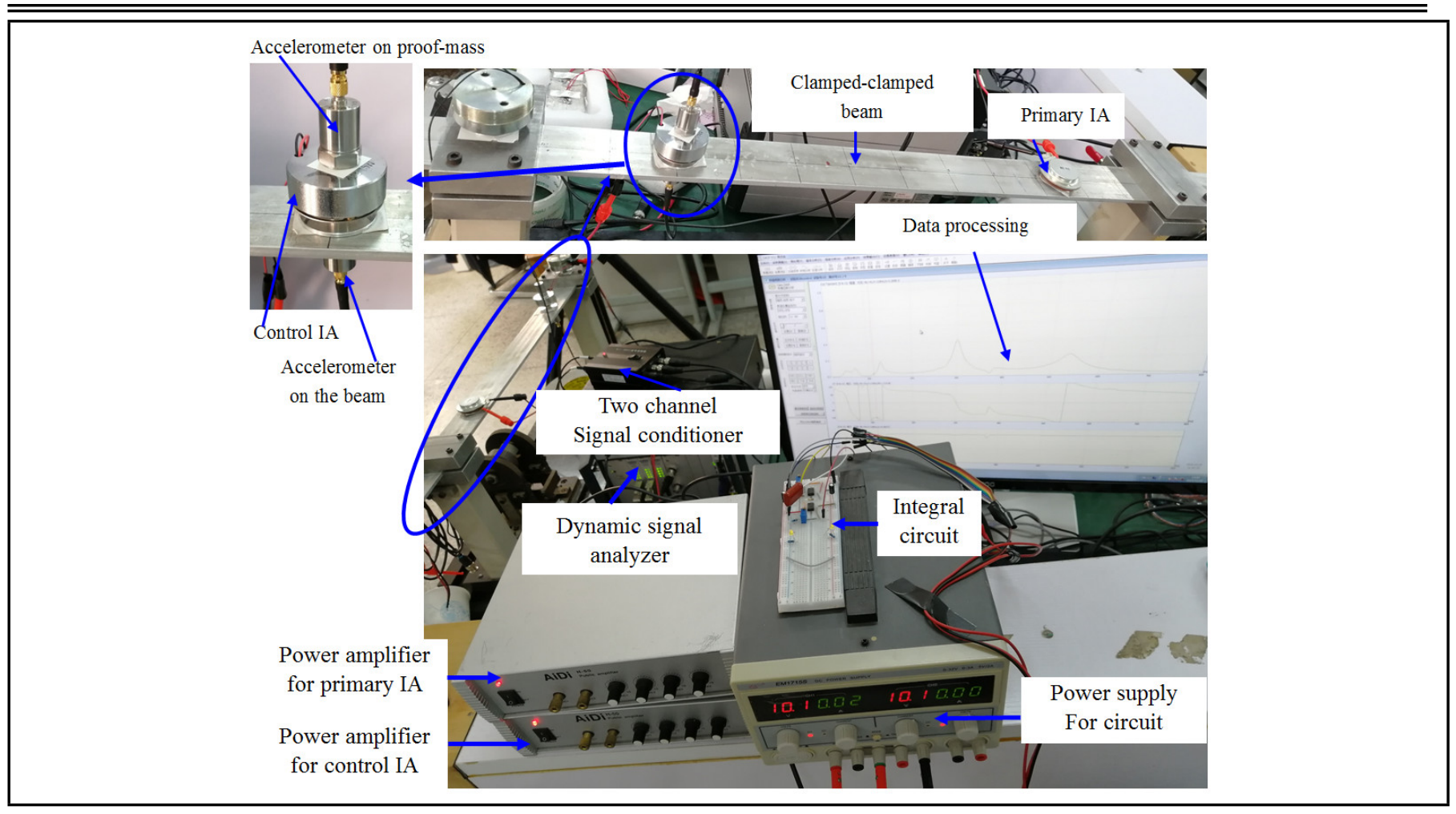

Figure 8. Experimental setup for velocity feedback control for a clamped-clamped beam by using the IA with virtual mass.

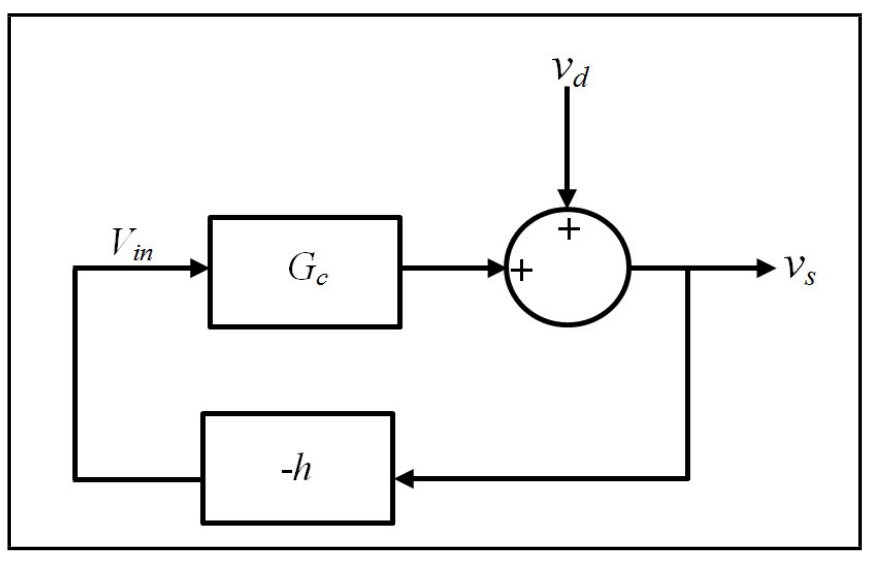

Figure 9. Block diagram of the velocity feedback control loop.

error sensor location can be expressed as

$$
v_{s}=V_{i n} G_{c}+v_{d}=-h v_{s} G_{c}+v_{d}
$$

where $V_{i n}=-h v_{s}$ was the control voltage and $h$ was the fixed feedback control gain. $G_{c}=\frac{v_{s}}{V_{i n}}$ was the open-loop FRF between the error sensor velocity $v_{s}$ and the control input voltage $V_{i n} . v_{d}$ was the velocity at error sensor location due to primary excitation source.

From Eq. (14), it can be found that the responses at the error sensor location with feedback control loop was expressed as

$$
v_{s}=\frac{v_{d}}{\left(1+h G_{c}\right)} .
$$

The objective of the controller in this study was to minimize the velocity at sensor location under certain stability margin. Notice that the practical sensor-actuator pair is not perfectly dual and collocated, ${ }^{4,9}$ it means that the velocity feedback control loop in this study is not unconditionally stable. Furthermore, the open-loop FRF $G_{c}$ can be modified under different virtual masses (or negative acceleration feedback gains). The open-loop FRFs $G_{c}$, between the voltage input to the control IA and the integrated beam-accelerometer outputs under different virtual masses were first discussed. The measured Bode and Nyquist plots of the open-loop FRFs are shown in Figs. 10 and 11 , respectively.

The effect of adding the virtual mass on the IA's natural frequency and the stability of the system can be found in both Bode and Nyquist plots. The first natural frequency of the beam $(100 \mathrm{~Hz})$ was arranged to be closed to the natural frequency of the passive IA $(99.5 \mathrm{~Hz}$, without virtual mass). Notice that the passive IA acted as a tuned vibration absorber at this situation, ${ }^{23,24}$ the mode splitting phenomenon can be found in Fig. 10. For open-loop FRF without virtual mass case, the peak at $77.5 \mathrm{~Hz}$ was associated with the lowest mode of the coupled IA-beam structure and has a $180^{\circ}$ phase shift in this case. It means that the control gain was very limited because a significant circle on the left-hand side of the Nyquist plot appears, as presented by the solid line in Fig. 11. However, when the proof-mass acceleration feedback loop was closed with feedback gains of 0.5 and 3 , the control IA's natural frequency was reduced to $80 \mathrm{~Hz}$ and $47.5 \mathrm{~Hz}$, respectively. Then the peak associated with the lowest mode was changed to $70 \mathrm{~Hz}$ and $47.5 \mathrm{~Hz}$, respectively. The circles on the left hand side of the Nyquist plot were reduced significantly when the control IA's natural frequency was reduced. Much higher velocity feedback gains were used. It means that the control system successfully attenuated the structural vibration when the virtual mass (proof-mass acceleration feedback loop) was introduced in the control IA.

The velocity responses measured at the control location of the beam, normalized to the input voltage of the primary excitation IA are presented in Fig. 12. From Fig. 12, it can be 

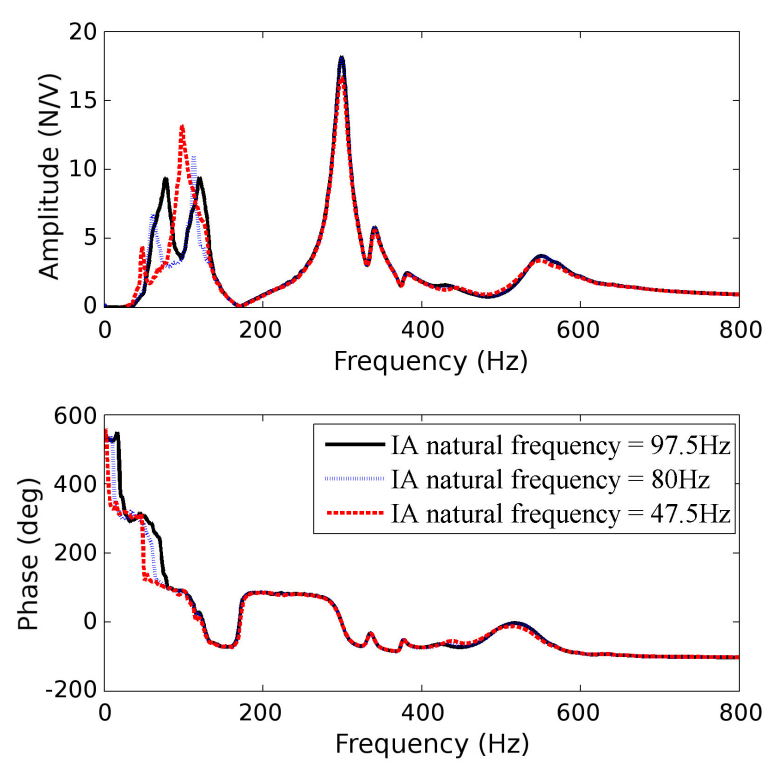

Figure 10. Bode plots of of the open-loop FRFs under different proof-mass acceleration feedback gains.

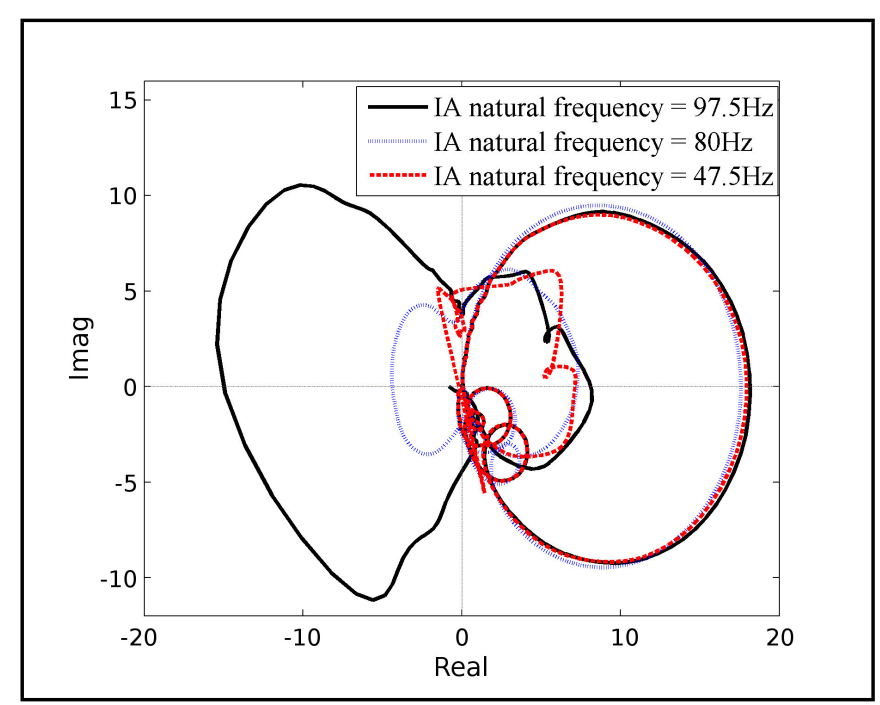

Figure 11. Nyquist plots of of the open-loop FRFs under different proof-mass acceleration feedback gains.

found that the passive IA acted as a tuned vibration absorber on the beam. The first mode of the beam was split into two new resonances at about $77.5 \mathrm{~Hz}$ and $120 \mathrm{~Hz}$ after mounting the passive IA without any feedback action, as presented by the dotted line in Fig. 12. Furthermore, the amplitudes of the 2nd and 3rd modes were also slightly reduced.

Then the control IA's natural frequency was shifted down to $80 \mathrm{~Hz}$ by using proof-mass acceleration feedback with gain $g_{p}$ $=0.5$. In this case, when the velocity feedback control loop was closed with the maximum stable gain, a peak with large amplitude at $70 \mathrm{~Hz}$ appeared, as presented by the dot-dashed line in Fig. 12. The reduction of vibration was very limited, where it can even become worse than that of passive case around $70 \mathrm{~Hz}$.

Finally, the control IA's natural frequency was moved to $47.5 \mathrm{~Hz}$ by using proof-mass acceleration feedback with gain $g_{p}=3$. In this case, when the velocity feedback gain approached the stability limit, about $8 \mathrm{~dB}$ vibration reduction was

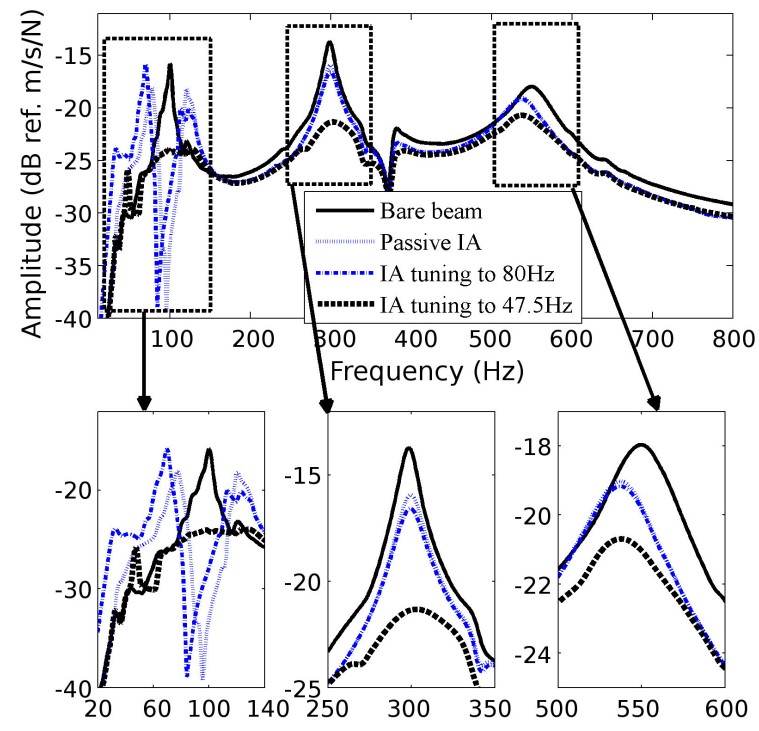

Figure 12. Measured velocity FRF at the error sensor location of the beam before and after control.

achieved for the first two modes at the error sensor location, the amplitude of the $3 \mathrm{rd}$ mode was also reduced $3.5 \mathrm{~dB}$, as presented by the dashed line in Fig. 12. However, at $47.5 \mathrm{~Hz}$, there was also some control enhancement of the vibration, due to the positive feedback in this frequency region caused by the phase response of the control IA.

\section{CONCLUSIONS}

In this paper, an IA with proof-mass acceleration feedback is used as a control unit for velocity feedback control approach. It is shown that the proof-mass acceleration feedback loop can be seen as a virtual mass, the effect of the virtual mass is to reduce the IA's natural frequency. The virtual mass of the IA proposed in this study uses simple acceleration control technology, and it is easy to implement because there is no need of the changes in IA physical design.

With an example of a clamped-clamped beam, the velocity feedback control experiments are performed to check the control performance of the proposed IA with virtual mass. When the IA's natural frequency is shifted from $80 \mathrm{~Hz}$ to $47.5 \mathrm{~Hz}$, the stability of the velocity feedback loop and control performance can be improved significantly. It means that the IA with appropriate proof-mass acceleration feedback gain can provide better control performance, because the IA's natural frequency is shifted to much lower frequency.

\section{ACKNOWLEDGEMENTS}

We wish to graciously thank the very perceptive reviewers for their careful review, suggestions, and helpful comments for this study. This work was sponsored by the National Natural Science Foundation of China (no. 51975266), the Natural Science Foundation of Jiangxi, China (no. 20192BAB206024), the Six Talent Peaks Project of Jiangsu Province, China (no. 2017-KTHY-036). 


\section{REFERENCES}

1 Haase T., Unruh O., Algermissen S. and Pohl M. Active control of counter-rotating open rotor interior noise in a Dornier 728 experimental aircraft, Journal of Sound and Vibration, 376, 18-32, (2016). https://dx.doi.org/10.1016/j.jsv.2016.04.038

2 Misol M. Full-scale experiments on the reduction of propeller-induced aircraft interior noise with active trim panels, Applied Acoustics, 159, 107086, (2020). https://dx.doi.org/10.1016/j.apacoust.2019.107086

3 Borgo M. D., Tehrani M. G. and Elliott S. J. Active nonlinear control of a stroke limited inertial actuator: Theory and experiment, Journal of Sound and Vibration, 465, 115009 , (2020). https://dx.doi.org/10.1016/j.jsv.2019.115009

4 Alujevic N., Senjanovic I., Catipovic I. and Vladimir N. The absence of reciprocity in active structures using direct velocity feedback, Journal of Sound and Vibration, 438, 251-256, (2019). https://dx.doi.org/10.1016/j.jsv.2018.09.035

5 Algermissen S. and Monner H. P. On the stability of decentralized AVC/ASAC for large-scale structures, Journal of Intelligent Materials Systems and Structures, 28 (16), 2255-2264, (2017). https://dx.doi.org/10.1177/1045389X16682843

6 Boulandet R., Michau M., Micheau P. and Berry A. Aircraft panel with sensorless active sound power reduction capabilities through virtual mechanical impedances, Journal of Sound and Vibration, 361, 2-19, (2016). https://dx.doi.org/10.1016/j.jsv.2015.09.042

7 Huyanan S. and Sims N. D. Vibration control strategies for proof-mass actuators, Journal of Vibration and Control, 13, 1785-1806, (2007). https://dx.doi.org/10.1177/1077546307080031

8 Zilletti M., Elliott S. J. and Gardonio P. Self-tuning control systems of decentralised velocity feedback, Journal of Sound and Vibration, 329 (14), 2738-2750, (2010). https://dx.doi.org/10.1016/j.jsv.2010.01.024

9 Diaz C. G. and Gardonio P. Feedback control laws for proof-mass electrodynamic actuators, Smart Materials and Structures, 16, 1766-1783, (2007).

10 Rohlfing J., Gardonio P. and Thompson D. J. Comparison of decentralized velocity feedback control for thin homogeneous and stiff sandwich panels using electrodynamic proof-mass actuators, Journal of Sound and Vibration, 330, 843-867, (2011). https://dx.doi.org/10.1016/j.jsv.2010.09.013

11 Camperi S., Tehrani M. G. and Elliott S. J. Parametric study on the optimal tuning of an inertial actuator for vibration control of a plate: theory and experiments, Journal of Sound and Vibration, 435, 1-22, (2018). https://dx.doi.org/10.1016/j.jsv.2018.07.048

12 Camperi S., Tehrani M. G. and Elliott S. J. Local tuning and power requirements of a multi-input multioutput decen- tralised velocity feedback with inertial actuators, Mechanical Systems and Signal Processing, 117, 689-708, (2019). https://dx.doi.org/10.1016/j.ymssp.2018.08.002

13 Alujevic N., Zhao G., Depraetere B., Sas P., Pluymers B. and Desmet W. $\mathrm{H}_{2}$ optimal vibration control using inertial actuators and a comparison with tuned mass dampers, Journal of Sound and Vibration, 333, 4073-4083, (2014). https://dx.doi.org/10.1016/j.jsv.2014.04.038

14 Olaru R., Arcire A., Petrescu C., Mihai M. M. and Gir$\tan \mathrm{B}$. A novel vibration actuator based on active magnetic spring, Sensors and Actuators A: Physical-Phys., 264, 1117, (2017). https://dx.doi.org/10.1016/j.sna.2017.07.041

15 Braghin F., Cinquemani S. and Resta F. A low frequency magnetostrictive inertial actuator for vibration control, Sensors and Actuators A: Physical-Phys., 180, 67-74, (2012). https://dx.doi.org/10.1016/j.sna.2012.03.015

16 Kras A. and Gardonio P. Velocity feedback control with a flywheel proof mass actuator, Journal of Sound and Vibration, 402, 31-50, (2017). https://dx.doi.org/10.1016/j.jsv.2017.05.001

17 Kras A. and Gardonio P. Active vibration control unit with a flywheel inertial actuator, Journal of Sound and Vibration, 464, 114987, (2020). https://dx.doi.org/10.1016/j.jsv.2019.114987

18 Zilletti M. Feedback control unit with an inerter proof-mass electrodynamic actuator, Journal of Sound and Vibration, 369, 16-28, (2016). https://dx.doi.org/10.1016/j.jsv.2016.01.035

19 Benassi L. and Elliott S. J. Active vibration isolation using an inertial actuator with local displacement feedback control, Journal of Sound and Vibration, 278, 705-724, (2004). https://dx.doi.org/10.1016/j.jsv.2003.10.065

20 Diaz I. M., Pereira E., Hudson M. J. and Reynolds P. Enhancing active vibration control of pedestrian structures using inertial actuators with local feedback control, Engineering Structures, 41, 157-166, (2012). https://dx.doi.org/10.1016/j.engstruct.2012.03.043

21 Rohlfing J., Elliott S. J. and Gardonio P. Feedback compensator for control units with proof-mass electrodynamic actuators, Journal of Sound and Vibration, 331 (15), 34373450, (2012). https://dx.doi.org/10.1016/j.jsv.2012.03.010

22 Diaz I. M. and Reynolds P. Robust saturated control of human-induced floor vibrations via a proof-mass actuator, Smart Materials and Structures, 18 (12), 125024, (2009).

23 Mao Q. and Huang S. Design of tuneable vibration absorber by using inertial actuator with proof-mass acceleration feedback, International Journal of Structural Stability and Dynamics, 19 (8), 1950087, (2019). https://dx.doi.org/10.1142/S0219455419500871

24 Elliott S. J., Rohlfing J. and Gardonio P. Multifunctional design of inertially-actuated velocity feedback controllers, Journal of the Acoustical Society of America, 131 (2), 1150-1157, (2012). https://dx.doi.org/10.1121/1.3672694 